\title{
Composición y estructura florística en fragmentos de bosques en los munici- pios de Yuscarán, Oropolí y Güinope, Departamento de El Paraíso, Honduras, C.A.
}

Thelma María Mejía Ordóñez

\section{Resumen}

En el Área Protegida "Reserva Biológica Yuscarán" los ecosistemas presentan una alta fragmentación quedando pocos fragmentos de su vegetación original. El objetivo fue determinar la composición y estructura florística en 5 fragmentos. Las mediciones se realizaron entre junio-agosto de 2011 , en parcelas de $20 X 50 \mathrm{~m}$ y $20 \mathrm{X}$ $20 \mathrm{~m}$. Se registró todos los individuos con DAP $\geq 2.5 \mathrm{~cm}$. Se calculó la densidad, dominancia, Índice de Diversidad. Se registró un total de 1339 individuos $\geq 2.5 \mathrm{~cm}$ de DAP, que representan 104 especies de 48 Familias y 81 Géneros. La abundancia y número de especies fue mayor en El Cerro Las Lechuzas. La Familia más numerosa fue la Fabaceae (leguminosas) con 19 especies. Las especies con mayor índice de valor de importancia fueron Pinus oocarpa, Pinus maximinoii, Mirospermum frutescen. Se registró un individuo con $90 \mathrm{~cm}$ de DAP de la especie endémica llex williamsii, en el Cerro El Zapotillo. Se registró 4 especies de preocupación especial Quercus bomelioides, Vitex gaumeri, Persea schiedeana y Guaiacum sanctum. El presente estudio es uno de los primeros en el área y se concluye que alberga una alta diversidad y que probablemente existen otras especies que falta registrar.

Palabras claves: Fragmentación, estructura florística, composición florística, abundancia, dominancia, diversidad, índice de valor de importancia.

Thelma María Mejía Ordóñez (mejiaordonez@gmail.com ) Escuela de Biología, UNAH y Fundación Yuscarán. Teléfono: 99903885 


\section{Introducción}

Las áreas protegidas de Honduras comprenden una superficie de 3 , $999,196.722 \mathrm{Ha}$ que representan un $29.55 \%$ de la superficie total del país $(112,482$ $\mathrm{km} 2$ ). Se distribuye esta superficie en 91 áreas protegidas (50 áreas prioritarias y 41 áreas no prioritarias). El Sistema Nacional de Áreas Protegidas de Honduras (SINAPH) está integrado por el conjunto de áreas protegidas legalmente declaradas bajo protección con fines de conservación de la diversidad biológica. Están clasificadas en 19 categorías de manejo, predominando las reservas biológicas y los parques nacionales (DAP, 2009).

Una de las actividades principales en el manejo de las áreas protegidas es la gestión y promoción de la investigación científica en temas sobre la biodiversidad, dinámica de ecosistemas, contaminación, entre muchas otras, con el objetivo de contar con información sobre riqueza de especies, tamaño de las poblaciones, la integridad ecológica del área, etc.

Sin embargo, la falta de investigaciones que informen sobre la estructura y composición de especies en las Áreas Protegidas de Honduras, trae como consecuencia la poca valoración de los fragmentos remantes, como refugios de la biodiversidad, especialmente la que presenta amenazas por causas de la pérdida de su hábitat como resultado de la fragmentación. La valoración de la vegetación natural remanente es de mucha importancia para el establecimiento de medidas de protección y conservación de nuestra biodiversidad.

La Fundación Yuscarán, con sede en la ciudad de Yuscarán, cabecera municipal del Departamento de El Paraíso, como responsable del manejo del Área Protegida Reserva Biológica Yuscarán y la Coordinación de Carrera de Biología, de la Universidad Nacional Autónoma de la UNAH, firmaron una Carta de Entendimiento para desarrollar un proyecto de investigación sobre el estado de la biodiversidad en la reserva, que se encuentra ubicada entre los municipios de Yuscarán, Oropolí y Güinope del Departamento de El Paraíso.

El Proyecto de Investigación enfocó sus estudios en los componentes de Flora y Fauna. En el componente de Flora se integró un estudio botánico y un estudio ecológico sobre la composición y estructura florística de las comunidades vegetales del sitio.

El objetivo principal del proyecto fue el levantamiento de información sobre la biodiversidad en la Reserva Biológica Yuscarán y su área de influencia en el marco del Corredor Biológico "La Unión", que integra los fragmentos de bosques 
y vegetación natural que están fuera del área protegida y que se ha comprobado funcionan como corredores biológicos y como objetivos especifico de la presente investigación se planteó caracterizar las comunidades vegetales en cuanto a su estructura y composición florística, evaluando la abundancia, dominancia, la importancia ecológica de las especies y la detección de especies de preocupación especial en los sitios evaluados.

\section{Descripción Del Área De Estudio}

\section{Ubicación geográfica de la Reserva Biológica Yuscarán}

El Área Protegida "Reserva Biológica de Yuscarán" (RBY), está ubicada en el Departamento de El Paraíso entre los municipios de Yuscarán, Güinope y Oropolí, está conformada por los cerros Monserrato, El Volcán y El Fogón, su altura máxima de 1991 msnm se encuentra en el Cerro El Volcán. La Reserva cubre un área total de 4,187 ha, de las cuales 1,562 ha corresponden a la zona núcleo y 2,625 ha a la zona de amortiguamiento (Fig.1).

El Municipio de Yuscarán del Departamento de El Paraíso, tiene una extensión de $336.3 \mathrm{Km} .{ }^{2}$ Su cabecera municipal se encuentra a 950 metros sobre el nivel del mar (msnm). Fue fundado en el año de 1730. Está ubicado a $65 \mathrm{Km}$. de Tegucigalpa, y es la Cabecera del departamento de El Paraíso. El Municipio de Yuscarán, limita al Norte con los Municipios de Morocelí y Potrerillos, al Sur con los Municipios de Oropolí y Alauca, al Este con los Municipios de Jacaleapa, San Matías y Alauca, y al Oeste con los Municipios de Güinope y San Antonio de Oriente. Políticamente está conformado por 19 aldeas. El Municipio de Yuscarán fue decretado Monumento Nacional en el año de 1979, por sus construcciones antiguas que aún se encuentran en el casco urbano, además su topográfica caracteriza la irregularidad sus calles, senderos y la riqueza del paisaje urbano.

Durante los siglos XVIII y XIX la explotación minera fue el principal rubro económico, actualmente las boca-minas e instalaciones mineras abandonadas, son representantes de esta actividad en la que se explotó la riqueza de la zona, primero por los españoles y luego por compañías norteamericanas. El municipio de Yuscarán es de vocación forestal, pero su principal actividad es la agricultura de cultivos tradicionales a pequeña escala como el maíz, frijol, café, hortalizas; esta actividad se complementa con el manejo de ganado vacuno y otras especies como gallinas y cerdos. También forma parte de la actividad económica algunas microempresas dedicadas a la siembra de ornamentales como las rosas, elaboración de cera liquida, desinfectantes jabón de zabila. 
El Municipio de Oropolí del Departamento del El Paraíso, tiene una extensión de $159 \mathrm{Km} .{ }^{2}$ Su cabecera municipal se encuentra a 550 msnm y se caracteriza por la presencia de bosque seco en la mayor parte de su área. Limita al Norte con el Municipio de Yuscarán, al Sur con los Municipios de San Lucas y San Antonio de Flores, al Este con el Municipio de Alauca, y al Oeste con el Municipio de Güinope. Políticamente está conformado por 11 aldeas. El Municipio de Oropolí es de vocación agropecuaria y la mayor parte de su población se dedica principalmente a la agricultura de cultivos especialmente granos básicos y hortalizas.

El Municipio de Güinope del Departamento de El Paraíso, se encuentra ubicado a unos $26 \mathrm{Km}$ al Sur del Valle El Zamorano sobre la carretera hacia la ciudad de El Paraíso. Posee una extensión territorial de $203.85 \mathrm{Km} 2$, con una altitud promedio entre los 700 y 1,250 msnm. Limita al Norte con el Municipio de San Antonio de Oriente del departamento de Francisco Morazán, al Sur con el Municipio de San Lucas del departamento de El Paraíso, al Este con los Municipios de Oropolí y Yuscarán del departamento de El Paraíso y al Oeste con el Municipio de Maraita del departamento de Francisco Morazán.

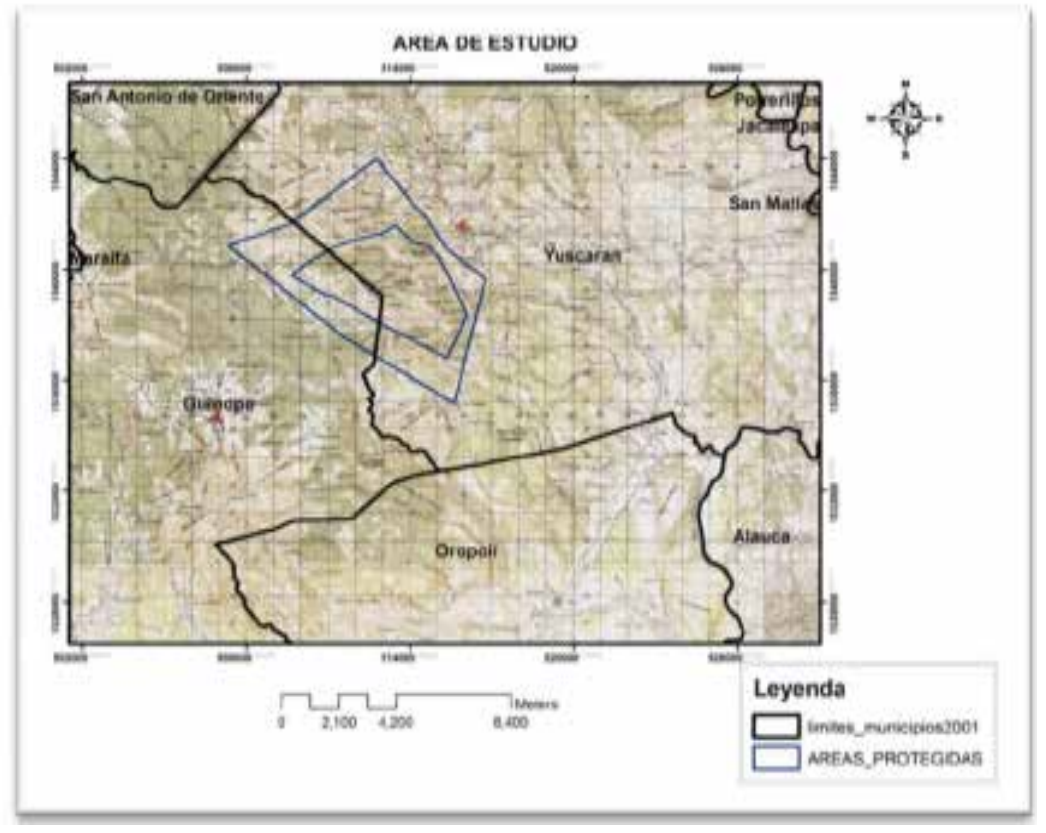

Figura 1. Mapa de ubicación geográfica de la Reserva Biológica Yuscarán, mostrada en líneas azules sobre la hoja cartográfica 


\section{Caracterización Biofísica}

\section{Geología}

Basados en el Mapa Geológico de Honduras (IGN), la RBY está formada por rocas volcánicas terciarias y sedimentarias de tipo riolítico y andesítico, rocas sedimentarias derivadas de rocas volcánicas y lavas de Riolitas, andesita y basalto. La formación Matagalpa es parte de la geología del área en la cual domina la andesita, basalto y sedimentos piroclásticos asociados.

\section{Geomorfología}

Se encuentra ubicada en una posición fisiográfica montañosa con grandes variaciones de elevación, las cuales forman fuertes depresiones o derrapes y áreas fuertemente escarpadas e inclinadas. Existen pequeños valles intermontanos con topografía irregular y de pendientes leves o suaves, la elevación sobre el nivel del mar varía entre 800 y 1991 metros.

\section{Suelos}

En el Área se presentan dos series de suelos la Serie Mulule y la Serie Yoro. En la serie Mulule el relieve es ondulado con pendientes entre 30 y $65 \%$, son suelos poco profundos, bien drenados y moderadamente ácidos, con una textura franco arenoso. Los suelos de esta serie son de vocación forestal. En la Serie Yoro el relieve es fuertemente ondulado, con pendientes comprendidas entre 25 y $7 \%$. Son suelos profundos, ácidos y desarrollados sobre cenizas volcánicas con una textura franco arenoso en los dos primeros horizontes y arcilloso areno en los demás.

\section{Hidrología}

La red hidrográfica de la RBY está formada al Norte por el rio La Montaña, las quebradas de Bachan, Quebrada Grande y Quebrada Piedra de la Aurora; al Sur por las Quebradas del Miguel, Las Calderas, Las Lomas, El Zaray y El Zapote, todas son afluentes del rio La Pita.

La Reserva Biológica Yuscarán (RBY) es la principal fuente abastecedora de agua que beneficia a los habitantes de varias comunidades de los Municipios de Yuscarán, Oropoli y Guinope. En el Municipio de Yuscarán abastece a la ciudad de Yuscarán, a las Aldeas de El Robledal, Agua Zarca, Chagüite Sur, Bachán, Ocotal, La Crucita y La Cidra. Al Norte la fuente de agua es abastecida por El Río La 
Montaña, la Quebrada de Bachán, la Quebrada Grande y la Quebrada Piedra de la Aurora, aquí también se favorece I Valle de El Zamorano.

Al sur de la RBY se benefician de la fuente agua algunas comunidades del Municipio de Güinope, en esta zona la fuente de agua es nutrida por la Quebrada de La Laguna, Quebrada Zapote y Quebrada El Horno. El Municipio de Oropolí también se beneficia con las aguas que le proporcionan las quebradas del Maguey, Las Calderas, Las lonas, El Zaray y El Zapote, todas ellas afluentes del río La Pita, que abastecen los sistemas de riego de diferentes comunidades.

\section{El Clima}

La precipitación promedio anual de la zona se registra con 1,562 mm, distribuida en dos épocas bien marcadas de seis meses cada una. Los promedios anuales varían según la altitud entre $1600 \mathrm{~mm}$ en las partes más bajas y $2000 \mathrm{~mm}$ en las partes más altas de la reserva.

Se registra una temperatura promedio anual de $21.81^{\circ} \mathrm{C}$ variando desde $21^{\circ} \mathrm{C}$ en elevaciones de $1000 \mathrm{msnm}$ a los $10^{\circ} \mathrm{C}$ en los picos más altos. En enero se registra el promedio más bajo $\left(19.6^{\circ} \mathrm{C}\right)$ y en los meses de abril y mayo los valores promedios más altos $\left(23.6^{\circ} \mathrm{C}\right.$ y $23.7^{\circ} \mathrm{C}$ respectivamente).

La evapotranspiración potencial (ETP) registra valores menores que la precipitación anual, los valores mensuales oscilan entre 91.8 y $141.0 \mathrm{~mm}$ y los valores más bajos se presentan en los meses de septiembre y noviembre $(91.8$ y $96.9 \mathrm{~mm}$ respectivamente), el valor más alto se presenta en el mes de abril con 141.0.

La humedad relativa promedio anual es de $73.5 \%$, presenta un rango promedio de 58.3 a $89.2 \%$. El valor más bajo se presenta en Febrero (58.3\%) y el valor más alto en el mes de septiembre (89.2\%). El promedio de humedad relativa en época seca es de $43.8 \%$ y para la lluviosa de $56.2 \%$.

\section{La Fauna}

En la RBY se han registrado especies de preocupación especial por su situación de amenaza en la disminución de sus poblaciones, debido a la destrucción del hábitat, al consumo de su carne a la caza para el uso de pieles, entre estas especies podemos mencionar el quetzal (Pharomacrus mocinno) al venado cola blanca (Odocoileus virginianus) al chancho de monte y al gato de monte. No se conoce la situación actual de las poblaciones de estas y otras especies de fauna presentes en la reserva. 


\section{Los Ecosistemas Vegetales}

Según el Mapa de Ecosistemas Vegetales de Honduras (AFE/COHDEFOR-BM, 2001), en el área de estudio se han identificado 4 ecosistemas vegetales: Bosque Tropical Siempreverde Estacional Latifoliado Montano Superior, Bosque Siempreverde Estacional Mixto Montano Inferior, Bosque Siempreverde Estacional Aciculifoliado Montano Inferior, Arbustal deciduo latifoliado de tierras bajas (Figura 2).

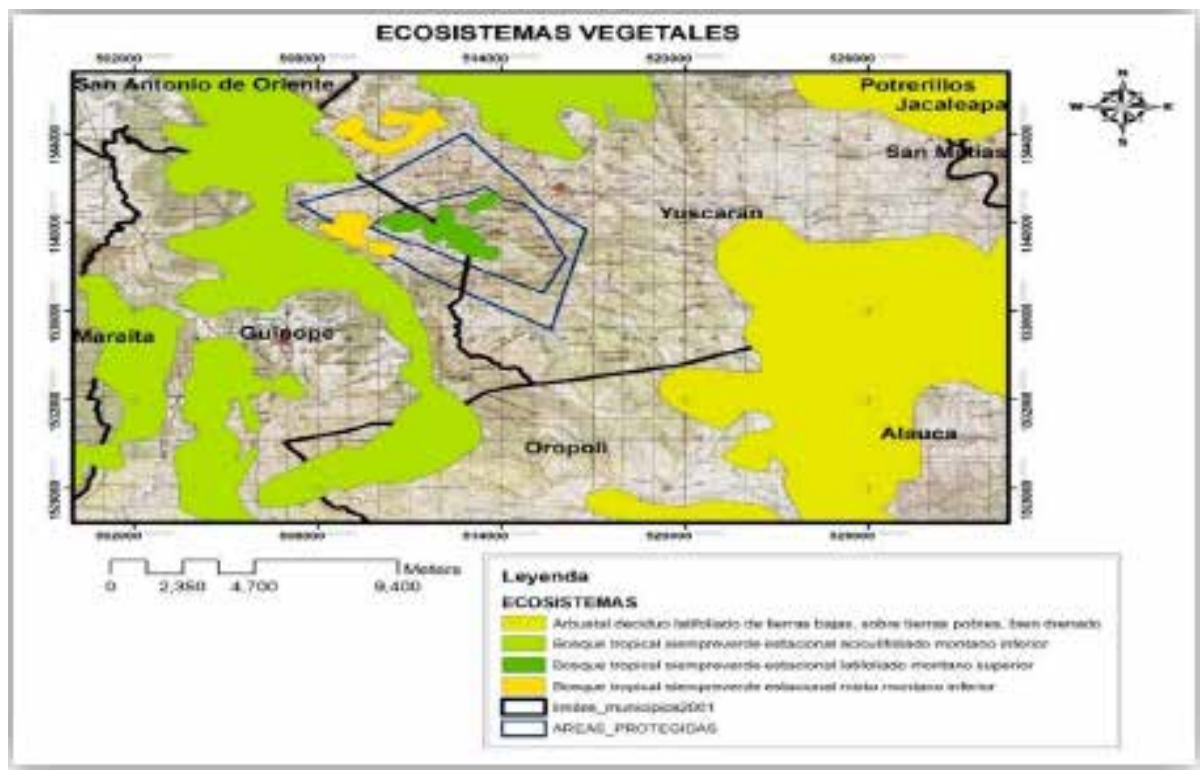

Figura 2. Mapa de Ecosistemas vegetales del área de estudio

\section{Metodología}

Se seleccionó 6 sitios de muestro considerando la presencia de remanentes de bosques que representan los ecosistemas vegetales del área de estudio (Figura 3).

Se estableció 11 parcelas temporales de diferentes tamaños: 6 de 20 $X 50 \mathrm{~m}, 5$ de $20 \times 20 \mathrm{~m}$ y se hizo dos evaluaciones sin parcela. El Cuadro No. 1 muestra la información de los sitios evaluados. 
El levantamiento de los datos de campo se realizó en el periodo de junio a agosto de 2011.

Cuadro 1. Sitios de muestreo, tamaño de la parcela, coordenadas de ubicación geográfica y código del sitio.

\begin{tabular}{|c|c|c|c|c|c|}
\hline \multirow[t]{2}{*}{ GIRA } & \multirow[t]{2}{*}{ NOMBRE DEL SITIO/NUMERO DE MUESTRA } & \multicolumn{2}{|c|}{$\begin{array}{l}\text { COORDENADAS } \\
\text { (UTM) }\end{array}$} & \multirow[t]{2}{*}{$\begin{array}{c}\text { ALTURA } \\
\text { (msnm) }\end{array}$} & \multirow[t]{2}{*}{$\begin{array}{l}\text { CODIGO } \\
\text { SITIO }\end{array}$} \\
\hline & & $x$ & $\mathrm{Y}$ & & \\
\hline 1 & $\begin{array}{l}\text { SITI0 1: GRANADILLA, YUSCARAN } \\
\text { MUESTRA 1: La Torera, por la Laguna, Parcela } \\
20 \times 50 \mathrm{~m}\end{array}$ & 511848 & 1540479 & 1731 & 1GRA1 \\
\hline \multirow[t]{5}{*}{2} & SITIO 2: EL TAMARINDO, YUSCARAN & & & & \\
\hline & $\begin{array}{l}\text { MUESTRA 2: El Cerro, parcela } 20 \times 20 \mathrm{~m} \\
\text { MUESTRA 3: Bosque de Galeria, Quebrada cerca }\end{array}$ & 525995 & 1534739 & 571 & 2TAM2 \\
\hline & $\begin{array}{l}\text { de la Escuela, Parcela } 20 \times 20 \mathrm{~m} \\
\text { SITIO 3: LA ESPERANZA, OROPOLI }\end{array}$ & 525752 & 1534483 & 475 & 2TAM3 \\
\hline & MUESTRA 4: Potrero arbolado, sin parcela & 520536 & 1525822 & 500 & 3ESP4 \\
\hline & MUESTRA 5: Bosque de Galeria, sin parcela & 520830 & 1525983 & 448 & 3 ESP5 \\
\hline \multirow[t]{4}{*}{3} & $\begin{array}{l}\text { SITI0 4: EL PERICON, YUSCARAN } \\
\text { MUESTRA 6: Cerro Ojo de Agua, Bosque Pino- } \\
\text { Encino, parcela } 20 \times 50 \mathrm{~m} \\
\text { MUESTRA 7: Cerro La Eminencia, parcela }\end{array}$ & 515768 & 1536722 & 1362 & 4PER6 \\
\hline & $\begin{array}{l}20 \times 50 \mathrm{~m} \\
\text { MUESTRA 8: Bosque pino de la bajura, Parcela }\end{array}$ & 515082 & 1536584 & 1456 & 4PER7 \\
\hline & $\begin{array}{l}20 \times 20 \mathrm{~m} \\
\text { MUESTRA 9: Bosque pino de la bajura, Parcela }\end{array}$ & 517297 & 1535797 & 945 & 4PER8 \\
\hline & $20 \times 30 \mathrm{~m}$ & 516713 & 1536027 & 1042 & 4PER9 \\
\hline \multirow[t]{3}{*}{4} & $\begin{array}{l}\text { SITI0 5: GUINOPE } \\
\text { MUESTRA 10: Montaña El Zapotillo, parcela }\end{array}$ & & & & \\
\hline & $20 \times 20 \mathrm{~m}$ & 505928 & 1530852 & 1894 & 5GUl10 \\
\hline & MUESTRA 11: Cerro EI Volcan, parcela $20 \times 50 \mathrm{~m}$ & 511565 & 1540019 & 1820 & 5 GUl11 \\
\hline \multirow[t]{3}{*}{5} & SITIO 6: EL RODEO, YUSCARÁN & & & & \\
\hline & $\begin{array}{l}\text { MUESTRA 12: Las Barrancas, Parcela } 20 \times 20 \mathrm{~m} \\
\text { MUESTRA 13: Cerro Las Lechusas, Parcela }\end{array}$ & 524777 & 1535579 & 560 & 6ROD12 \\
\hline & $20 \times 50 \mathrm{~m}$ & 523889 & 1538084 & 511 & 6ROD13 \\
\hline
\end{tabular}




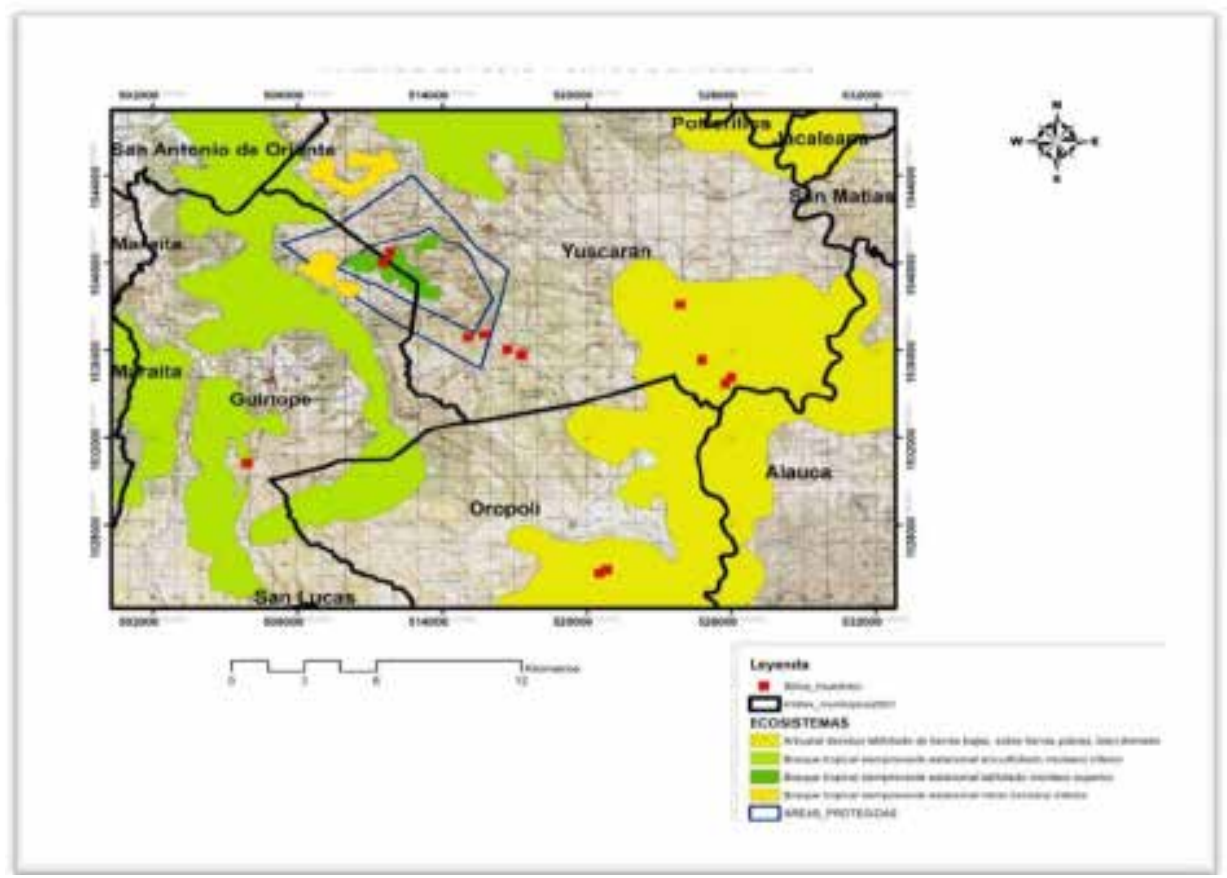

Figura 3. Mapa del área de estudio donde se muestra los sitios de muestreo en puntos rojos, ubicados en los tres diferentes ecosistemas del área de estudio

En cada una de las parcelas se evaluó todos los individuos con un DAP $\geq$ $2.5 \mathrm{~cm}$, no se incluyó epifitas, hierbas y lianas. Se colectó una muestra botánica cuando el individuo estaba estéril y tres copias de individuos fértiles (flores, frutos 0 ambos), fueron numeradas de acuerdo a la parcela correspondiente. La altura fue estimada de manera aproximada por observación. La identificación de las especies se realizó en el Herbario TEFH de la Escuela de Biología-UNAH.

Para el análisis de la estructura vegetal de cada remanente, se calculó el Índice de Valor de Importancia (IVI). El Índice de Valor de Importancia es un método para evaluar la dominancia y caracterizar una comunidad vegetal. Fue creado por Curtis y Mclntosh (1951), bajo la premisa de que "la variación en la composición florística es una de las características más importantes que deben ser determinadas en el estudio de una vegetación". El Índice de Valor de Importancia (IVI) es un indicador de la importancia fitosociológica de una especie, dentro de una comunidad.

EI IVI es uno de los índices más utilizados en el análisis de ecosistemas tropicales (Lamprecht, 1990; Plonczak, 1993; Dezzeo et al., 2000; García et al, 
2010). Su principal ventaja es que es cuantitativo y preciso; no se presta a interpretaciones subjetivas. Además, suministra una gran cantidad de información en un tiempo relativamente corto. Soporta análisis estadísticos y es exigente en el conocimiento de la flora. El método no sólo proporciona un índice de importancia de cada especie, también aporta elementos cuantitativos fundamentales en el análisis ecológico, como la densidad y la biomasa (por especie y por parcela). Este último, es un carácter básico para interpretar la productividad de un sitio, lo cual depende en gran medida del bio-clima y de los recursos edáficos.

En el IVI, la dominancia se evaluó el área basal o superficie que ocupa un tallo que posee un diámetro o circunferencia determinado. Este parámetro tiene una relación directa con la cobertura o la biomasa.

El Índice de Importancia (li), es una simplificación del IVI de Curtis y Mclntosh (1951) donde se obvia la Frecuencia, es muy susceptible al tamaño de las sub-parcelas. Por consiguiente, el Índice de Importancia (li) para este estudio se calculó con la siguiente ecuación:

$$
\mathrm{li}=\mathrm{A} \%+\mathrm{Dom} \%
$$

Donde:

$$
\begin{aligned}
& \text { - } \mathrm{A} \%=\text { abundancia relativa } \\
& \text { - } \mathrm{Dom} \%=\text { dominancia relativa }
\end{aligned}
$$

Diversos trabajos han utilizado este indicador, entre ellos conviene mencionar a Ramírez (1995), en los Llanos Occidentales de Venezuela; Ramírez-García et al. (1998), en manglares de México y Dewalt et al. (2003), en Panamá. Tiene las mismas ventajas del IVI. Pero, el li parece ser más adecuado en parcelas pequeñas, donde el tamaño de las sub-parcelas hace inconveniente el cálculo de la frecuencia.

\section{Resultados}

\section{Composición y estructura florística}

Los resultados de la composición florística del área de estudio a nivel todos los sitios evaluados, muestran que se registró un total de 1339 individuos, distribuidos en 48 Familias, 81 Géneros y 104 especies identificadas, el mayor número se encontró en el sitio de muestreo llamado Cerro Las Lechuzas, de El Rodeo, Yuscarán, en una parcela de $20 \times 50 \mathrm{~m}$. La Familia más numerosa resulto ser la 
Fabaceae (leguminosas) con 19 especies, seguida de la Fagaceae (los robles) con 8 especies, la Rubiaceae (la familia del café) con 5 especies. Un total de 26 individuos no fueron identificados taxonómicamente debido a que no se pudo obtener una muestra botánica fértil, algunas veces por la altura de los individuos otras debido a su estado fenológico estéril. (Cuadro No.2).

En cuanto a la estructura de las comunidades, la distribución diamétricas mostro que 344 individuos están agrupados en un DAP (Diámetro a la altura del Pecho, tomándolo a $1.30 \mathrm{~m}$ ) entre 2.5 y 4.99 , 445 en un DAP entre 5 y 9.99 y 550 en un DAP mayor de 10.

En el Bosque Nublado se evaluaron 3 parcelas: 1 en Yuscarán y 2 en Güinope, en la primera se encontró 24 especies, en la segunda 13 y en la tercera 20, esta última corresponde a la muestra del Cerro El Volcán, un sitio muy bien conservado pues mostro arboles con DAP mayores de $100 \mathrm{~cm}$ y abundantes helechos arborescentes de más de 2 metros de altura (Fig. 4).

El bosque de pino y pino-encino que se encuentra en el Sitio 4 El Pericón, Yuscarán, fue evaluado mediante 4 muestras, aquí se midieron 260 individuos, dentro de los cuales se encontró 4 especies diferentes de robles y dos especies de pinos. Aproximadamente el $50 \%$ de los individuos se encuentra agrupados en la Clase Diamétrica de mayor de $10 \mathrm{~cm}$.

\begin{tabular}{|c|c|c|c|c|c|c|c|c|c|c|}
\hline \multirow{3}{*}{ Sitio } & \multirow{3}{*}{$\begin{array}{l}\text { Número } \\
\text { Familias }\end{array}$} & \multirow{3}{*}{$\begin{array}{l}\text { Número } \\
\text { Géneros }\end{array}$} & \multirow{3}{*}{$\begin{array}{l}\text { Número } \\
\text { Especies }\end{array}$} & \multicolumn{6}{|c|}{ Numero de especies e individuos por clases diamétricas } & \multirow{3}{*}{$\begin{array}{c}\text { Número } \\
\text { Total } \\
\text { Individuos }\end{array}$} \\
\hline & & & & \multicolumn{2}{|c|}{ 25-4.99 } & \multicolumn{2}{|c|}{$5-9.99$} & \multicolumn{2}{|c|}{$>10$} & \\
\hline & & & & $\begin{array}{c}\text { Num. } \\
\text { Esp. }\end{array}$ & $\begin{array}{l}\text { Num. } \\
\text { Ind. }\end{array}$ & Num. Esp. & $\begin{array}{l}\text { Num. } \\
\text { Ind. }\end{array}$ & $\begin{array}{l}\text { Num. } \\
\text { Esp. }\end{array}$ & $\begin{array}{l}\text { Num. } \\
\text { Ind. }\end{array}$ & \\
\hline 1GRA1 & 13 & 14 & 24 & 18 & 93 & 17 & 79 & 13 & 72 & 244 \\
\hline 2TAM2 & 7 & 12 & 17 & 12 & 102 & 10 & 39 & 3 & 11 & 152 \\
\hline 2TAM3 & 13 & 16 & 17 & 13 & 40 & 10 & 41 & 7 & 22 & 103 \\
\hline 3ESP4 & 5 & 6 & 6 & 0 & 0 & 2 & 6 & 5 & 40 & 46 \\
\hline 3ESP5 & 12 & 16 & 19 & 2 & 2 & 8 & 16 & 15 & 61 & 79 \\
\hline 4PER6 & 4 & 5 & 7 & 1 & 1 & 4 & 10 & 7 & 71 & 82 \\
\hline 4PER7 & 2 & 4 & 4 & 0 & 0 & 1 & 1 & 4 & 33 & 34 \\
\hline 4PER8 & 4 & 4 & 4 & 1 & 2 & 3 & 16 & 2 & 18 & 36 \\
\hline 4PER9 & 3 & 3 & 3 & 2 & 7 & 2 & 15 & 3 & 29 & 51 \\
\hline 5GUl10 & 12 & 12 & 13 & 5 & 13 & 5 & 42 & 10 & 33 & 88 \\
\hline 5GU111 & 9 & 10 & 20 & 1 & 3 & 7 & 70 & 17 & 53 & 126 \\
\hline $6 \mathrm{ROO} 12$ & 12 & 15 & 18 & 8 & 40 & 11 & 42 & 13 & 25 & 107 \\
\hline $6 R 0013$ & 18 & 25 & 25 & 17 & 41 & 20 & 68 & 21 & 82 & 191 \\
\hline TOTAL & 48 & 81 & 130 & 68 & 344 & 69 & 445 & 84 & 550 & 1339 \\
\hline
\end{tabular}

Cuadro 2. Composición y estructura florística en los sitios de muestreo 

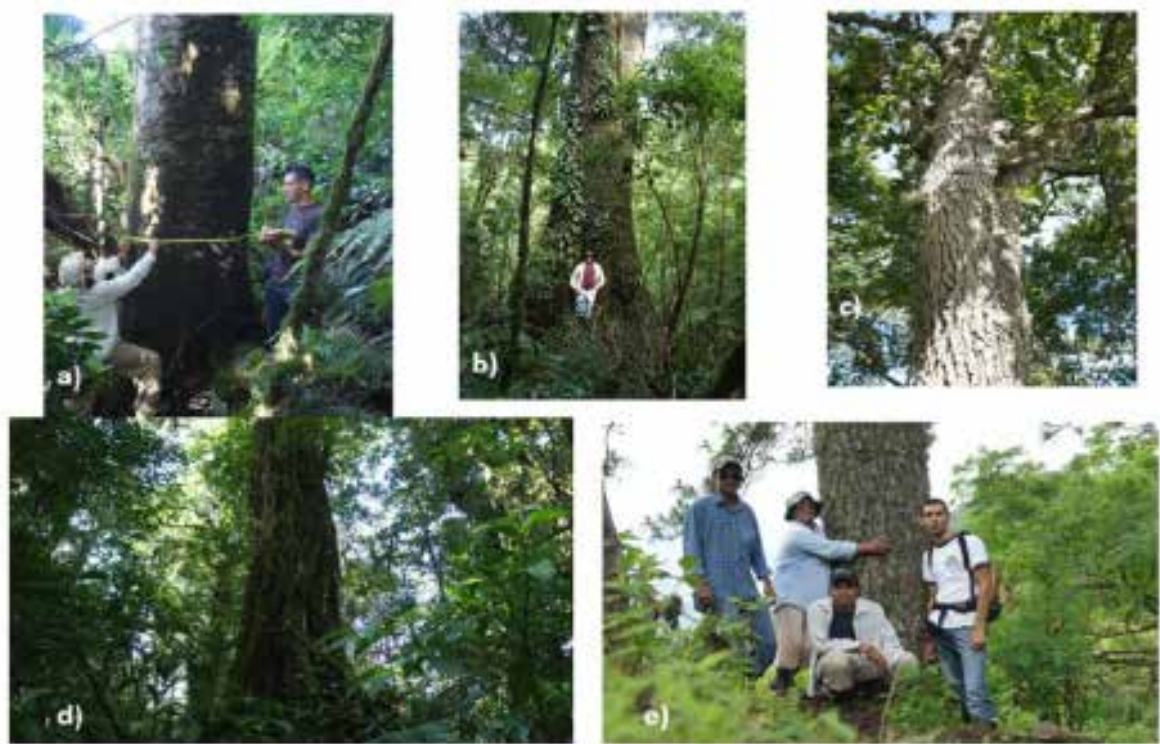

Figura 4. Aquí se muestra algunos individuos que definen la estructura de la vegetación en los sitios de muestreo, por sus prominentes características: a) individuo de más de $90 \mathrm{~cm}$ de DAP y arriba de los 25 metros de altura (aproximada), del Sitio Cerro El Zapotillo; en b) y d) dos individuos de más de 40 metros de altura y un DAP mayor de $100 \mathrm{~cm}$, estos del Cerro El Volcán; en d) vemos un individuo del genero Quercus de más de 25 metros en el bosque de pino encino de El Pericón y en e) un individuo de Pinus maximinoi de $85 \mathrm{~cm}$ dimétricos con más de 30 metros de altura, en el área del Cerro La Eminencia.

\section{Índice de Importancia (li)}

La especie dominante con el más alto valor de importancia fue Pinus Oocarpa con 153.9 del Sitio El Pericón, Yuscarán; 4 especies con alto índice representan al Bosque Seco y para el Bosque Nublado tenemos a la Psichotria panamensis, con un índice de 73.2, esta especie resulto muy abundante en el sotobosque (Cuadro No. 3). 


\begin{tabular}{|c|c|c|c|c|c|c|c|c|c|c|c|}
\hline \multirow{2}{*}{ Nombre común } & \multirow{2}{*}{ Nombre Cientifico } & \multicolumn{9}{|c|}{ Sitios de Muestreo } & \multirow{2}{*}{ Ecosistema } \\
\hline & & 2 TAM2 & 2 TAM 3 & 3ESP5 & 4PER6 & 4PERT & 4PER8 & APERT & 5GUl11 & $6 R 0012$ & \\
\hline Chapema negra & Mrospermum futescens & 1334 & & & & & & & & & Bosque seco \\
\hline Jifilicuao & Bursera simlaba & & 89.8 & & & & & & & & Bosque seco \\
\hline Almendro de río & Andra nermis & & & 77.5 & & & & & & & Bosque seco \\
\hline Pino & Pinus occapa & & & & 57.1 & & 137.6 & 1539 & & & Bosque de pino-encino \\
\hline Pino & Prus maxinixi & & & & & 1026 & & & & & Bosque de pino \\
\hline Sin nombre cmun & Psichotra pramamenss & & & & & & & & 73.2 & & Bosque Latifoliado \\
\hline Huesito & Allophyes racenosus & & & & & & & & & 58.1 & Bosque seco \\
\hline
\end{tabular}

Cuadro 3. Índice de Importancia (li), para las especies

\section{Especies de Preocupación Especial}

Es muy importante mencionar que en el presente estudio se registró una especie endémica Ilex williamsii Standl., de la Familia Aquifoliaceae, se encontró un individuo de $90 \mathrm{~cm}$ dimétricos y de aproximadamente 30 metros de altura en la parcela que se evaluo en el Cerro El Zapotillo del Municipio de Güinope. (Fig.5 a).

Además se encontró 4 especies de Preocupación Especial, en las categorías de "Vulnerable" y "En Peligro" según de Lista Roja de la UICN. Quercus bomelioides, ubicada en la categoría de "Vulnerable", fue encontrada en dos sitios: en Güinope se midió 6 individuos en una parcela de 20 X 20 m con un promedio de DAP de $46 \mathrm{~cm}$ y en Granadilla 1 individuo en una parcela de $20 \times 50 \mathrm{~m}$, con un promedio de DAP de $27 \mathrm{~cm}$. Vitex gaumeri en categoría "En peligro", se encontró en un bosque de galería, un individuo con un promedio de DAP igual a $11 \mathrm{~cm}$ y una altura aproximada de $4 \mathrm{~m}$. Persea schiedeana, aguacatillo, en categoría "Vulnerable", fue encontrado 1 individuo en el bosque nublado del Cerro el Volcán, con un DAP promedio de $68 \mathrm{~cm}$., en una parcela de $20 \times 50 \mathrm{~m}$. Finalmente Guaiacum sanctum en la categoría "En Peligro" se midió un individuo con $9 \mathrm{~cm}$ de DAP y una altura aproximada de 3 metros, en una parcela de $20 \times 50 \mathrm{~m}$., el guayacán es altamente explotado se extrae la corteza y se cortan los arboles a muy temprana edad, sin embargo es destacable que en el sitio donde se encontró se observó una alta regeneración de pequeños arbolitos de más o menos 1 metro de altura (Cuadro No.4 y Figura 5). 


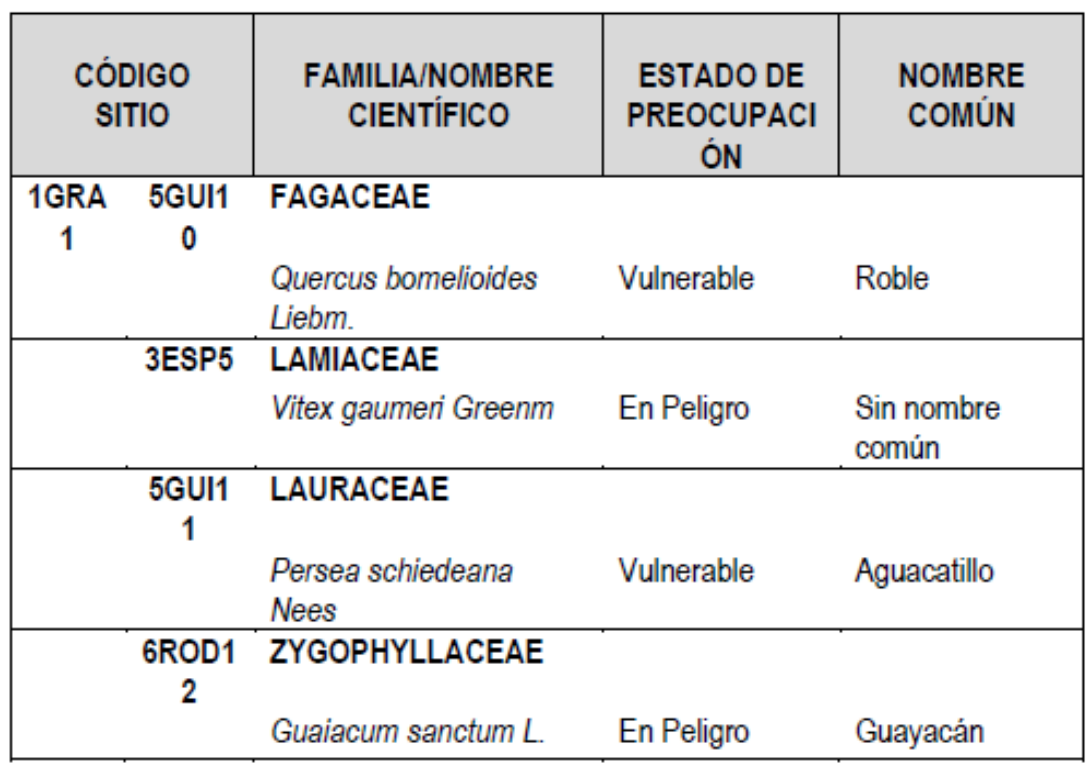

Cuadro 4. Especies de Preocupación Especial
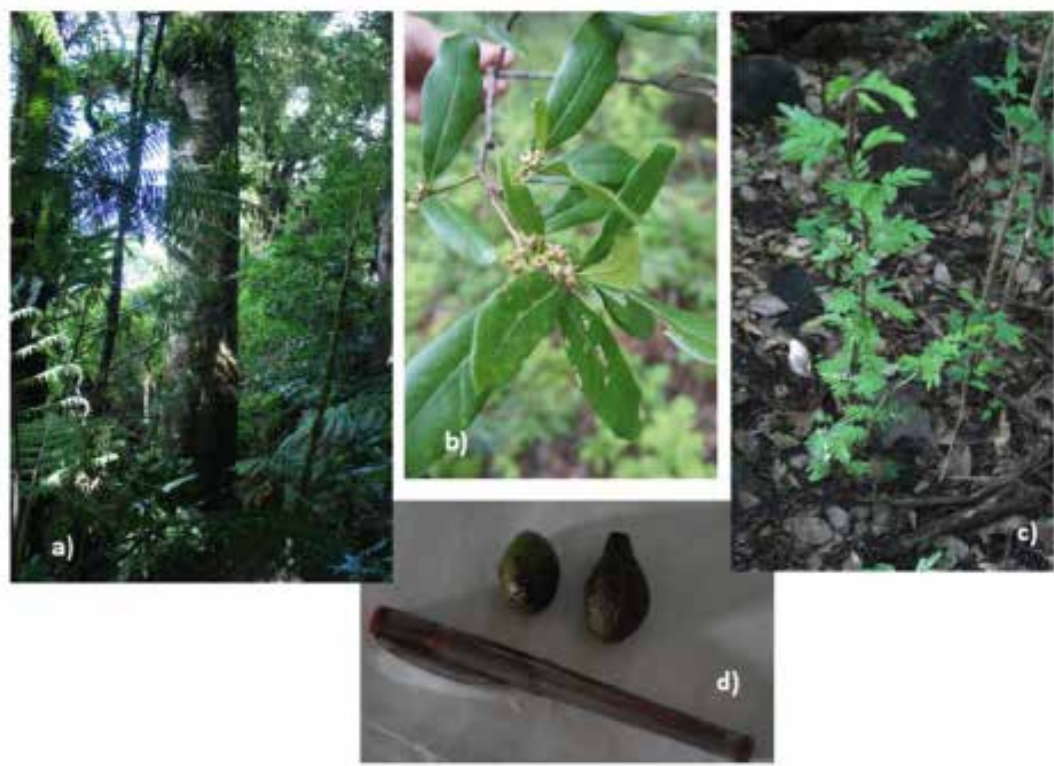

Figura 5. Especies de Preocupación Especial: a) llex williamsii, b) Quercus bomelioides,

c) Guaiacum sanctum y d) frutos de Persea schiedeana 


\section{Discusión}

\section{Composición y estructura florística}

Aquí es importante destacar que la Muestra 1, ubicada en La Torera, Granadilla, Yuscarán, fue la que más individuos registro (244), sin embargo la Muestra 13, del Cerro Las Lechuzas, la segunda en abundancia de individuos (191), fue la que mayor número de familias géneros y especies registro, esta muestra es representante del Bosque Seco, lo cual nos indica que los remanentes de este tipo de bosque aun guardan una alta diversidad.

Los fragmentos de bosques evaluados, albergan una alta riqueza de especies, y su estructura diamétrica muestra que aproximadamente un $40 \%$ de los individuos presentaron un diámetro a la altura de pecho menor o igual de $10 \mathrm{~cm}$ y el $60 \%$ presentaron un diámetro mayor o igual a 10 , dentro de estos últimos se registró individuos de 80,90 y hasta $120 \mathrm{~cm}$ dimétricos, con alturas de más de 30 metros Figura 4).

\section{Índice de Importancia (li)}

El Índice de Importancia considera la Dominancia Relativa que se calcula en base al Área Basal de cada una de las especies, según el DAP de los individuos. El total de área basal para todos las muestras fue de $34.5 \mathrm{~m} 2 /$ ha, para un total de área muestreada de $0.76 \mathrm{ha}$ (7,600 metros), este bajo valor se justifica por el alto porcentaje de individuos con DAP menor a $10 \mathrm{~cm}$. La mayor Área Basal $(6.7 \mathrm{~m} 2 / \mathrm{ha})$ se encontró en la muestra del bosque nublado del Cerro El Volcán, aquí encontramos 4 individuos de la especie Quercus cortesii con más de $100 \mathrm{~cm}$. De DAP

\section{Especies de Preocupación Especial}

El área de estudio es un sitio que a pesar de presentar una alta fragmentación, brinda refugio a especies de preocupación especial en las categorías de "vulnerable" y en "peligro", lo mismo que a una especie endémica, esto la hace poseedora de un alto valor ecológico que merece el establecimiento de programas de conservación que incluya la evaluación y monitoreo de las poblaciones de las especies mencionadas. 


\section{Conclusiones}

En el área de estudio aún se encuentran fragmentos de bosques que albergan una gran cantidad de especies vegetales arbóreas, se registró 130 especies, aunque 26 de ellas no se identificación taxonómicamente.

Se encontró 4 especies vegetales de preocupación especial en el bosque nublado, bosque seco y bosque pino encino.

Una de las desventajas que presentan los fragmentos es su aislamiento de los parches más grandes y el tamaño, algunos están completamente rodeados por terrenos de cultivos.

Una ventaja que tienen algunos de los fragmentos es su ubicación, sus pendientes inclinadas, esto no los hace aptos para cultivo o ganadería.

\section{Agradecimientos}

Quiero expresar mi profundo agradecimiento a todas las personas que estuvieron involucradas de una u otra manera, en la realización de la presente investigación científica, tanto con el apoyo económico, logístico, asistencias, alimentación, hospedaje, compañerismo, amistad y toda la clase de ayuda necesaria para desarrollar y culminar un trabajo de tanta importancia para el apoyo de la investigación, vinculación con la sociedad, la academia y la conservación y manejo de nuestros recursos naturales.

\section{Referencias Bibliográficas}

- Braun-Blanquet, J. 1979. Fitosociología. H. Blume. Madrid. España. 820 p.

- Curtis, J. y R. Mcintosh. 1951. An upland forest continuum in the prairie-forest border region of Wisconsin. Ecology 32: 476-496.

- DAP. Octubre 2009. Manual de Procedimientos para la elaboración de Planes de Manejo en las Áreas Protegidas del SINAPH. Tegucigalpa, HN.

- Dezzeo, N., P. Maquirino, P. Berry y G. Aymard. 2000. Principales tipos de bosque en el área de San Carlos de Río Negro, Venezuela. Scientia Guaianae 11: $15-36$.

- Dewalt, S., S. Maliakal y J. Denslow. 2003. Changes in vegetation structure and composition along a tropical forest chronosequence: implications for wildlife. Forest Ecology and Management 182(1-3): 139-151. 
- García, C., C. Suarez \& M. Daza. 2010. Estructura y diversidad florística de dos bosques naturales (Buenos Aires, Depto. Cauca, Colombia). Facultad de Ciencias Agropecuarias. Vol 8 (1). 9 pp.

- Lamprecht, H. 1990. Silvicultura en los trópicos. Agencia de Cooperación Técnica Alemana (GTZ). Eschborn, Alemania. 335 p.

- Lozada D., J. R. (2010). Consideraciones metodológicas sobre los estudios de comunidades forestales. Revista Forestal Venezolana, Año XLIv, Volumen 54(1): 77-88.

- Matteucci, S y A. Colma. 1982. Metodología para el estudio de la vegetación. Secretaría General de la Organización de los Estados Americanos. Washington, USA. 168 p.

- Ramírez-García, P., J. López-Blanco y D. Ocaña. 1998. Mangrove vegetation assessment in the Santiago River Mouth, Mexico, by means of supervised classification using LandsatTM imagery. Forest Ecology and Management 105 (13): 217-229.

- San Martín, J., A. Espinosa, S. Zanetti, E. Hauenstein, N. Ojeda \& C. Arriagada. 2008. Composición y estructura de la vegetación epífita en un bosque primario de Olivillo (Aextoxicon punctatum $R$. et $P$.) en el sur de Chile. Ecología Austral. Asociación Argentina de Ecología. 18:1-11. 12 pp. 